\title{
Toxicity Evaluation of Acrylamide on the Early Life Stages of the Zebrafish Embryos (Danio rerio)
}

\author{
Hattie Spencer ${ }^{1}$, Joseph Wahome1, Mary Haasch ${ }^{2}$ \\ ${ }^{1}$ Department of Natural Sciences and Environmental Health, Mississippi Valley State University, Itta Bena, Mississippi, USA \\ ${ }^{2}$ National Center for Natural Products Research, Environmental Toxicology Research Program, University of Mississippi, Oxford, \\ Mississippi, USA \\ Email: hspencer@mvsu.edu
}

How to cite this paper: Spencer, H., Wahome, J. and Haasch, M. (2018) Toxicity Evaluation of Acrylamide on the Early Life Stages of the Zebrafish Embryos (Danio rerio). Journal of Environmental Protection, 9, 1082-1091.

https://doi.org/10.4236/jep.2018.910067

Received: June 20, 2018

Accepted: September 4, 2018

Published: September 7, 2018

Copyright $\odot 2018$ by authors and Scientific Research Publishing Inc. This work is licensed under the Creative Commons Attribution International License (CC BY 4.0).

http://creativecommons.org/licenses/by/4.0/

\begin{abstract}
Acrylamide is a chemical used mainly in industrial applications and the treatment of drinking and wastewater, making it easy to enter aquatic ecosystems. There are few studies known about the toxicity of acrylamide to aquatic organisms which have shown evidence of a number of histopathological effects. To assess the effects of acrylamide to freshwater fish, Zebrafish (Danio rerio) embryos were exposed to serial concentrations of acrylamide $(0,100$, 300 , and $500 \mathrm{mg} / \mathrm{L}$ ) to investigate the acute toxicity effects on teleost embryogenesis. Embryos less than 24 hrs old were exposed under static non-renewal conditions for ten days or until hatching. The toxic endpoints evaluated include: egg/embryo viability, hatchability, and morphologi$\mathrm{cal} /$ developmental anomalies during organogenesis. The acute toxicity test resulted in a 48 h-LC50 of $585 \mathrm{mg} / \mathrm{L}$ for egg viability. Exposure of embryos significantly reduced hatchability and larval survival, in a concentration dependent manner. Dimethyl sulfoxide (DMSO) was used as a solvent carrier to permeate the uptake of acrylamide through the chorion membrane. No significant damages or complications were observed in embryos exposed to DMSO. At $500 \mathrm{mg} / \mathrm{L}$, the highest test concentration, the survival of embryos was greatly reduced within $24 \mathrm{hrs}$ of exposure. The lower test, $100 \mathrm{mg} / \mathrm{L}$, produced a significant number of developmental anomalies to the Zebrafish that included dorsal tail flexure, severe pericardial edema, facial and cranial defects and decreased heartrate $(40 \mathrm{bpm})$. Premature hatching of embryos and developmental arrest was observed in all concentrations. The severity of these anomalies was concentration-dependent and resulted in low survival rate and high frequency of malformations. These results indicate that acrylamide is teratogenic and provide support for sub-lethal toxicity testing using Zebrafish embryos.
\end{abstract}




\section{Keywords}

Acrylamide, Toxicity, Zebrafish, Malformation, Embryonic Development

\section{Introduction}

Acrylamide is a chemical intermediate used in the production and synthesis of polyacrylamide [1] [2]. It is a synthetic chemical compound commonly used in many branches of industry. The largest use for polyacrylamide is treating municipal drinking water and wastewater to remove suspended solids. The polymer is used to remove suspended solids from industrial wastewater before discharge, reuse, or disposal. Recent discoveries have shown that people are exposed to small amounts of acrylamide through its presence in some starchy foods cooked at high temperatures [3]. Effects of acrylamide on human health and the environment depend on how much acrylamide is present and the length and frequency of exposure [4]. The most important environmental contamination results from the use of acrylamide in soil grouting and drinking-water contamination by the use of polyacrylamide flocculants that contain residual acrylamide monomer [5] [6] and from acrylamide-based sewer grouting and wastepaper recycling [7] [8].

The EPA's Toxics Release Inventory reported environmental releases of $8,797,482 \mathrm{lb}$ of acrylamide from 42 facilities in the US, $99.9 \%$ of which was released to underground injection wells [9]. Levels detected of $400 \mathrm{mg} / \mathrm{l}$ in well-water in Japan had been contaminated from a grouting operation and residual acrylamide concentrations for water-treatment plants ranged from 0.5 to 600 ppm [10] [11]. Acrylamide monomers may not be removed in many water treatment processes and remain stable for more than two months in tap water and can become a potential source of pollution.

Laboratory animals exposed to acrylamide exhibited a decrease in glutathione level and in the activity of glutathione-S-transferase on the brain and liver. The inhibition of glutathione-S-transferase by acrylamide, which catalyzed conjugation with glutathione, may lead to the accumulation of the monomer and it enhanced neurotoxicity [12]. In addition, laboratory studies in animals have also shown that exposure to acrylamide can induce cancer, genetic damage and adverse effects on reproduction and development. Several studies have shown acrylamide to have a moderate acute toxicity effect to aquatic organisms [13]. At present, little is known about the toxic effects of acrylamide in fishes. Acrylamide toxicity study conducted in two species of aquatic macroinvertebrates and three species of fish demonstrated that acrylamide was moderately toxic aquatic organisms [14]. There was also histopathological changes and correlation with genotoxicity and metabolic alterations when Carassius auratus hepatopancreas (goldfish) was exposed to acrylamide [15].

Since acrylamide could be released into water systems, there is a need to un- 
derstand and evaluate its impact on the early life stage development of aquatic organisms. Fish is an important component of the ecosystem and the early life stages of fish have been found to be more sensitive to pollutants than adult [16]. Proliferating embryonic tissue can be particularly sensitive to damage from reactive metabolites generated by xenobiotic metabolizing enzymes [17].

In this study the zebrafish, (Danio rerio) was the model used to assess the developmental processes and toxicological effects of acrylamide on developing embryo. The zebrafish is idea due to ease of maintenance, translucent and non-sticky eggs, and a short reproduction cycle [18]. A non-static renewal bioassay was used to assess hatchability, and development. Furthermore, the study will determine if acrylamide is teratogenic to the developing embryo.

\section{Materials and Experimental Methods}

\subsection{Test Material}

Acrylamide $\left(\mathrm{C}_{3} \mathrm{H}_{5} \mathrm{NO}\right)$-Lot No. 148571-25G, Purity 97\%, and Dimethyl Sulfoxide,CAS No. 67-68-5, was both purchased from Sigma-Aldrich Chemical Company (St. Louis, MO). Dimethyl Sulfoxide (DMSO) was used as a solvent carrier. Instant Ocean Sea Salt was used for preparing embryo rearing medium (egg water) and was obtained from Aquarium Systems (Vernon, CT). The embryos were obtained from a continuous culture at the University of Mississippi, (Oxford, MS) Biology department. The water temperature was set at $27^{\circ} \mathrm{C}$ with a cycle of 14 hours light: 10 hours dark. Eggs were collected within 5 hours of spawning and examined microscopically for fertilization and stage development. Embryos were transferred to a clean $20 \mathrm{ml}$ vials containing $15 \mathrm{ml}$ of rearing medium (60 $\mu \mathrm{g} / \mathrm{ml}), 15 \mathrm{ul}$ of DMSO and appropriate concentration of test chemical. The rearing medium used in this study consisted of the following: $10 \mathrm{~g} \mathrm{NaCl}, 0.3 \mathrm{~g}$ $\mathrm{KCl}, 0.4 \mathrm{~g} \mathrm{CaCl}_{2}$, and $1.63 \mathrm{~g} \mathrm{MgSO}_{4}$ in $1000 \mathrm{~mL}$ of deionized water. Normal hatching period for untreated zebrafish embryo was 56 hours after fertilization. Embryos, both treated and untreated, completing the hatching process were recorded daily. Hatched larvae were not fed due to the duration of the test. Fertilized eggs were examined daily to observe embryonic development, time point of lesion and abnormal development, developmental arrest and hatching success.

\subsection{Acute Exposure}

The study was conducted in a static non-renewal system. The rearing medium consisted of Instant Ocean Sea Salts (40 g) added to 1liter distilled water. The stock solution was diluted with rearing medium to the desired acrylamide concentrations of 100, 300 and $500 \mathrm{mg} / \mathrm{L}$. These concentrations are based on the high levels of acrylamide released in the effluents from treatment plants into the aquatic environments.

Twenty embryos (two replicates) were placed in $15 \mathrm{ml}$ of rearing medium and incubated at a temperature of $27^{\circ} \mathrm{C}$. Photoperiod was timer-controlled with 14 hours light and 10 hours dark. Each group of 20 embryos was examined daily for 
signs of early life stage toxicity. There were two control groups, a positive (rearing medium only) and vehicle control (DMSO plus rearing medium). DMSO made the embryo more sensitive to the uptake of acrylamide to reach sufficient concentrations needed for the exposure.

Dead and hatched embryos were counted and removed daily. Embryo developmental stage, time point of lesion and mortality was determined by microscopic examination. Egg mortality was based on the number of embryos that died prior to hatching and that was opaque in appearance. Embryos were exposed to concentrations until hatched.

\subsection{Statistical Analysis}

The EPA Probit analysis method was used to calculate the $\mathrm{LC}_{50}$ value and its $95 \%$ confidence intervals, from the $96-\mathrm{h}$ acute toxicity data. For the sub-acute experiments, descriptive statistics were applied to determine the mean values of each experimental data set. An analysis of variance (ANOVA, F-test) was performed to determine whether there were significant differences among these means. The Student t-test was used to compare each of the treated measurements with the control measurements, which included the DMSO vehicle control. The level of significance was set at $\mathrm{p}=0.05$ (95\% confidence level).

\section{Results and Discussion}

\subsection{Time Hatching and Egg Mortality}

The hatching success of non-exposed (control) and exposed zebrafish embryos are presented in Figure 1. The acute exposure of zebrafish embryos to acrylamide caused a significant decrease in hatching that correlated with increasing acrylamide concentrations. Embryos were exposed to acrylamide after fertilization at concentrations of 100,300 , and $500 \mathrm{mg} / \mathrm{L}$. Hatching for zebrafish embryos began at 56 hours where $100 \%$ of embryos hatched in the control group and $95 \%$ fry hatched in the vehicle control. No death was observed in the DMSO exposed embryos during the first 24 hours, however, the mean percentage of egg mortality for DMSO embryos were $5 \%$ for the remainder of development.

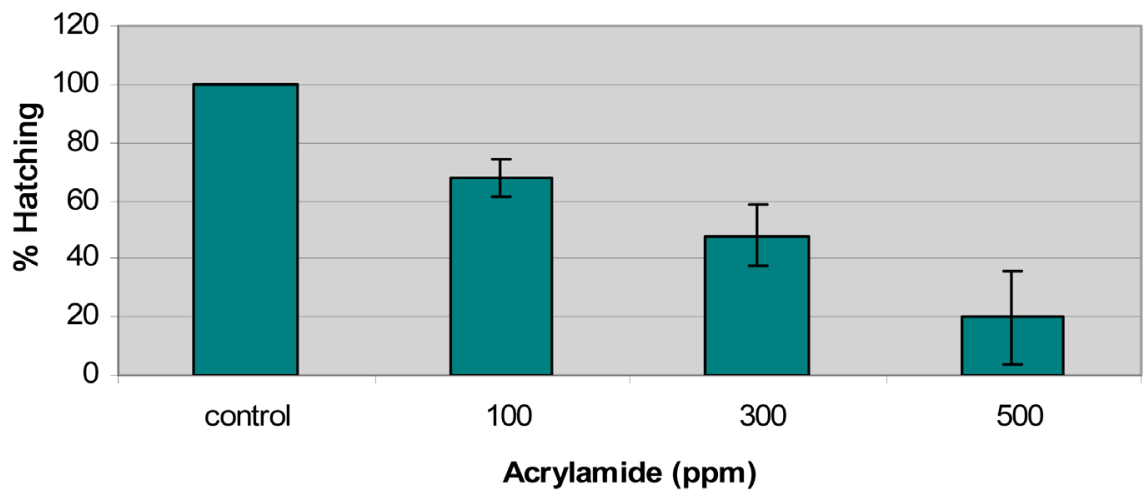

Figure 1. Effects of Acrylamide on hatching success of Zebrafish embryos at different concentrations. $\mathrm{p}=0.05$ ( $95 \%$ confidence level). $(\mathrm{N}=40$ per Acrylamide concentration). 
DMSO did not appear to have a negative effect on the development of the zebrafish during the hatching period. The non-exposed embryos showed normal signs of teleostean development based on the developmental series for the zebrafish embryo [19]. Hatching and egg mortality disparities were observed in all concentrations as well as delayed hatching and a reduction in the number of embryos that completed the hatching process. Egg mortality was shown to be concentration dependent. Death in exposed was observed within 24 hours and continued to increase throughout embryos development. Statistical analysis using the F-test (ANOVA) shows highly significant differences $(\mathrm{p}=0.01)$ in mean percentages of hatching among experimental groups. Therefore, it could be concluded that hatching was inversely correlated with acrylamide concentrations. Statistical analysis using the F test (ANOVA) shows highly significant difference among hatching experimental groups.

The diffusion of the acrylamide in the zebrafish embryo was extremely slow in which slow infusion of a chemical is a protective mechanism of the outer chorion and membrane systems that protect the embryo from chemical insult [20]. DMSO made the embryo more sensitive to the uptake of acrylamide to reach sufficient concentrations needed for the exposure.

\subsection{Teratogenic Effects of Acrylamide}

Characteristics signs of acrylamide early life stage toxicity in embryo are presented in Figure 2. The embryonic abnormalities were observed within 32 hours of exposure, indicating that acrylamide may be teratogenic. Major signs of acrylamide toxicity observed were severe pericardial and yolk sac edema, scoliosis (dorsal tail flexure), and cranial defects (sloping forehead). As illustrated, there was a concentration-response relationship with respect to the induction of each of these developmental abnormalities by acrylamide. The earliest adverse physiological effect produced by induced acrylamide toxicity on the developing zebrafish embryo was pericardial and yolk sac edema at a concentration of $100 \mathrm{ppm}$.

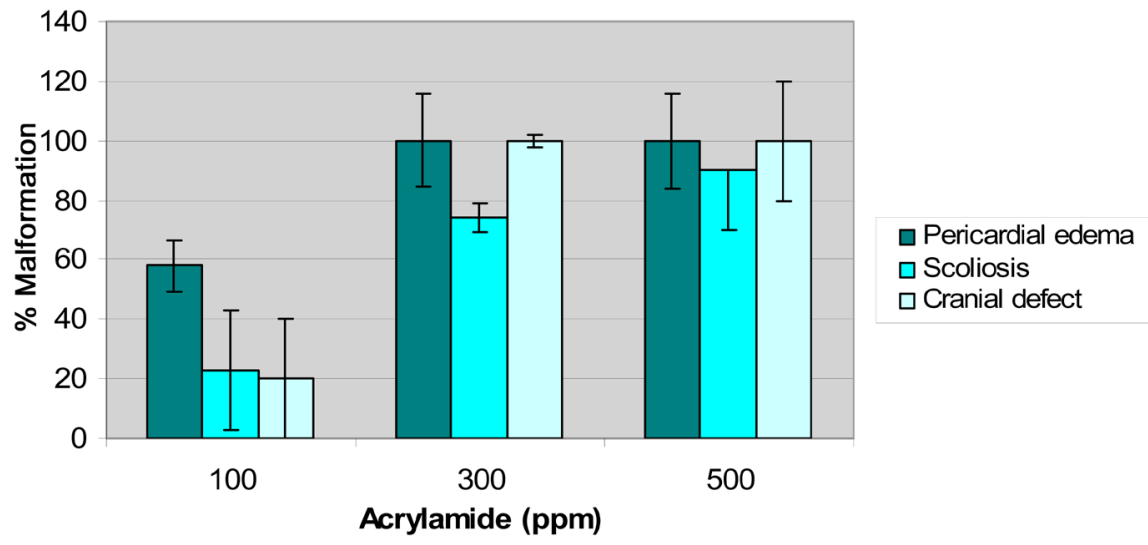

Figure 2. Percentage of developmental abnormalities in the zebrafish embryos exposed to acrylamide. $\mathrm{p}=0.05$ (95\% confidence level). $(\mathrm{N}=40$ per Acrylamide concentration). $(\mathrm{N}=$ 40 per Acrylamide concentration). 
For instance, $0.0,23,74$ and 90 percent of scoliosis malformations were observed in embryos exposed to 0 (Control), 100, 300 and 500 ppm of acrylamide, respectively.

\subsection{Effects of Acrylamide on Heartrate and Blood Flow in Zebrafish Embryo}

When exposed to acrylamide shortly after fertilization, zebrafish embryos exhibit reduced heartrate and blood flow by 32 hours post fertilization (hpf). The effects of acrylamide on the heartrate of the zebrafish embryo are shown in Figure 3. Data illustrated indicates a dose-response relationship related to the toxicity of acrylamide. All control embryos exhibit a normal heartrate throughout hatching. The average heart rate for control at $32 \mathrm{hr}$ was 142 beats per minutes (bpm). Within 32 hours, embryos began to show sign of stress. All treated embryos, began to show oscillation blood flow pattern associated with a decreased blood flow and heartrate in all concentrations after 32 and 48 hours of exposure, but more profound at 48 hrs. Upon 48 hours of exposure, the average percentage heartrate for embryos in each concentration were $146,83,70,40 \mathrm{bpm}$ at concentrations of $0,100,300$ and 500 ppm, respectively. Oscillation blood flow pattern usually occurred in conjunction with slow blood flow. Similar patterns of blood flow were also observed in the yolk sac in the vitelline vein of the embryo. After 48 hours of exposure, the vitelline vein became a broad band in the treated embryos. With an increase in concentration, the number of lesions (blood clots, hemorrhage, and cessation of blood flow) increased in treated embryos.

\subsection{Effects of Increasing Concentrations of Acrylamide on Malformation Development in Zebrafish Embryo}

The primary site of action of acrylamide in the zebrafish embryos was the profound effects on the circulatory and nervous systems. The earliest sign of acrylamide

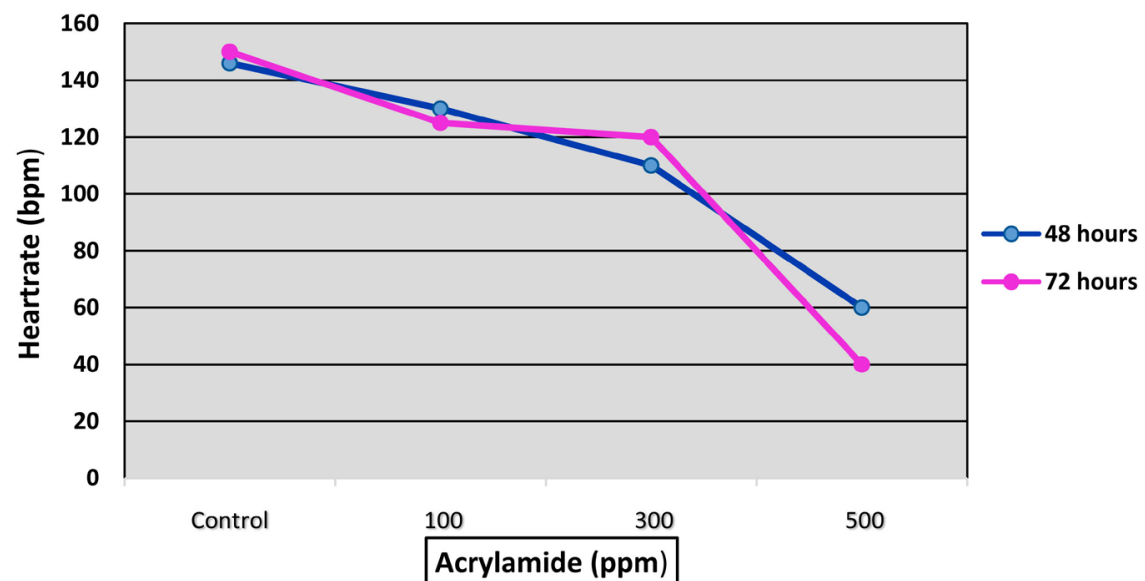

Figure 3. Effects of acrylamide on heartrate in the zebrafish embryo at 48 and 72 hours at different concentration. The heartrate in beats per minutes $(\mathrm{bpm})$ decreased from $\sim 146$ to $60 \mathrm{bpm}$ in 48 hours and from $\sim 150$ to $40 \mathrm{bpm}$ in 72 hours. $\mathrm{p}=0.05$ ( $95 \%$ confidence level). $(\mathrm{N}=40$ per Acrylamide concentration). 
induced toxicity after post-fertilization was the cardiovascular and nervous system. An increase in concentration resulted in an increase in the percentage abnormalities occurring in the zebrafish embryos. A normal control zebrafish embryo and fry shown in Figure 4(a) illustrates normal zebrafish embryonic and larvae development. A treated embryo and fry from (100 ppm) is shown in Figure 4(b) with moderate pericardial and yolk-sac edema, sloping forehead and scoliosis after induction of acrylamide toxicity, indicating that organogenesis may be the most sensitive stage of embryonic development. A severe form of pericardial edema and scoliosis was also observed at $300 \mathrm{ppm}$ (Figure 4(c)). The same effects were notable but more severe than observed in the $100 \mathrm{ppm}$ treated embryo. The embryo shown in Figure 4(d) reflects a greater degree of severity of the lesion cause by acrylamide exposure. After 72 hours, most embryos in this concentration were not hatched.

Acrylamide produce more severe forms of pericardial edema, cranial defect and scoliosis at $500 \mathrm{ppm}$ than seen at any other concentrations. The severity of these malformations also produced a high death rate in the embryo which indicates that acrylamide becomes increasingly fatal to embryos at higher concentrations. Pericardial edema gives rise to the collapse of the yolk sphere which prevents the heart from undergoing normal chamber formation [21].

Scoliosis of the spine was commonly observed in all concentrations. Scoliosis in fish is most likely caused by agents that act on the central nervous system, or the neuromuscular junctions [22]. Similar lesion observed in zebrafish exposed has been attributed to calcium depletion [23].

\section{Conclusion}

This study illustrated that acrylamide was acutely toxic to the development of the zebrafish embryo. The stage of embryonic development through the larval stage of a fish life is most sensitive to environmental contaminants. The exposure to sublethal concentrations of acrylamide resulted in a significant reduction in hatching, as well as the development of a number of morphological and physiological abnormalities in the zebrafish. Acrylamide exposure induces developmental

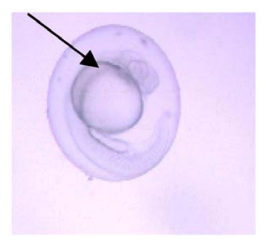

(a)

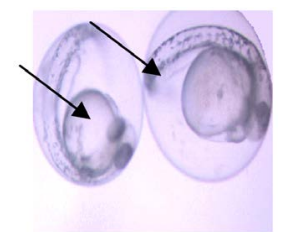

(b)

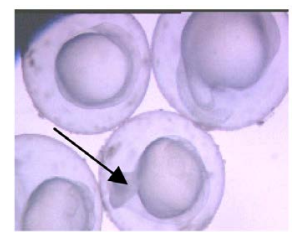

(c)

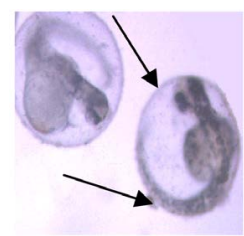

(d)

Figure 4. (a) Control embryos showing normal teleostean development; (b) Zebrafish embryo treated with 100 ppm acrylamide. Embryo and larvae showed signs of yolk-sac edema and dorsal curvature of the spine. Sloping forehead and deformed mouth was also evidence of acrylamide toxicity; (c) 300 ppm acrylamide treated embryos showing defect in head and trunk development and along with yolk-sac edema; (d) 500 ppm treated embryos showing delayed hatching with severe cranial deformation, scoliosis and yolk-sac edema (40× Magnification). 
abnormalities in the zebrafish embryos, which leads to developmental arrest and delayed hatching, especially in the highest concentration. The lowest tested concentration produced a significant number of developmental effects to the zebrafish embryo, including pericardial edema, dorsal tail flexure, cranial defect, and defects in heart morphology. The severity of these malformations also produced a high death rate in the embryo. These results indicated that acrylamide becomes increasingly fatal to embryos, reducing survival at higher concentrations when exposed during early development. In addition, the zebrafish is a good test model for assessing and screening the wide range of effect following exposure to acrylamide and the potential for risks to human health at low levels.

\section{Acknowledgements}

We thank Dr. Michael Smith for his expertise and assistant with this research and Dr. Ali Ishaque and Dr. Frank von Hippel for critically reading the manuscript. This research was financially supported by a grant from the National Institute of Child Health and Human Development (NICHD) from the National Institute of Health (NIH) grant number: GII HD 37065-05 and MBRS grant.

\section{Conflicts of Interest}

The authors declare no conflicts of interest regarding the publication of this paper.

\section{References}

[1] IARC, International Agency for Research on Cancer (1986) IARC Monographs on the Evaluation of the Carcinogenic Risk of Chemicals to Humans. Some Chemicals used in Plastics and Elastomers, 39, 403.

http://citeseerx.ist.psu.edu/viewdoc/download?doi=10.1.1.173.3428\&rep=rep1\&type $=\mathrm{pdf}$

[2] IARC, International Agency for Research on Cancer (1994) IARC Monographs on the Evaluation of Carcinogenic Risks to Humans. Some Industrial Chemicals, 60, 560 .

http://citeseerx.ist.psu.edu/viewdoc/download?doi=10.1.1.173.3428\&rep=rep1\&type $=\mathrm{pdf}$

[3] National Institute of Environmental Health Sciences (2004) NTP-CERHR Monograph on the Potential Human Reproductive and Developmental Effects of Acrylamide. Center for the Evaluation of Risks to Human Reproduction (CERHR). https://ntp.niehs.nih.gov/ntp/ohat/acrylamide/acrylamide_monograph.pdf

[4] U.S.EPA. (1994) U.S. Environmental Protection Agency. Office of Pollution Prevention and Toxics. Chemical in the Environment: Acrylamide. EPA 79-06-1, Washington DC.

[5] NSF (1988) Drinking Water Treatment Chemicals-Health Effects. Ann Arbor, MI, National Sanitation Foundation (Standard 60). http://www.who.int/water_sanitation_health/dwq/chemicals/acrylamide.pdf

[6] WHO (1985) Acrylamide. Environmental Health Criteria 49. World Health Organization, Geneva. http://www.iprev.it/uploads/media/ACRYLAMIDE.pdf

[7] Brown, L., Rhead, M.M., Bancroft, K.C.C. and Allen, N. (1980b) Model Studies of 
the Degradation of Acrylamide Monomer. Water Research, 14, 775-778. http://www.iprev.it/uploads/media/ACRYLAMIDE.pdf

[8] Brown, L., Rhead, M.M., Hill, D. and Bancroft, K.C.C. (1982) Rapid Screening Technique Utilizing High-Performance Liquid Chromatography for Assessing Acrylamide Contamination in Effluents. Analyst, 107, 749-754. http://www.iprev.it/uploads/media/ACRYLAMIDE.pdf

[9] TRI (2009) TRI Explorer Chemical Report. U.S. Environmental Protection Agency. http://www.epa.gov/triexplorer

[10] Igisu, H., Goto, I., Kawamura, Y., Kato, M., Izumi, D. and Kuroiwa, Y. (1975) Acrylamide Encephaloneuropathy Due to Well Water Pollution. Journal of Neurology, Neurosurgery, and Psychiatry, 38, 581-584.

[11] Howard, P.H. (1989) Acrylamide. In: Handbook of Environmental Fate and Exposure Data for Organic Chemicals, Vol 1, Large Production and Priority Pollutants, Lewis Publishers, Chelsea, MI, 13-19.

[12] Shanker, R. and Seth, P.K. (1986) Toxic Effects of Acrylamide in a Freshwater Fish, Heteropneustes fossilis. The Bulletin of Environmental Contamination and Toxicology, 37, 274-280.

[13] Kegley, S.E., Hill, B.R., Orme, S. and Choi, A.H. (2016) PAN Pesticide Database, Pesticide Action Network, North America, (Oakland, CA). http://www.pesticideinfo.org

[14] Krautter, G.R., Mast, R.W., Alexander, H.C., Wolf, C.H., Friedman, M.A., Koschier, F.J. and Thompson, C.M. (2009) Acute Aquatic Toxicity Tests with Acrylamide monomer and Macroinvertebrates and Fish. Environmental Toxicology and Chemistry, 5, 373-377. https://doi.org/10.1002/etc.5620050406

[15] Larguinho, M., Costa, P.M., Sousa, G., Costa, M.H., Diniz, M.S. and Baptista, P.V. (2013) Histopathological Findings on Carassius auratus Hepatopancreas upon Exposure to Acrylamide: Correlation with Genotoxicity and Metabolic Alterations. Journal of Applied Toxicology, 34, 12.

[16] McKim, J.M. (1977) Evaluation of Tests with Early Life Stages of Fish for Predicting Long-Term Toxicity. Journal of the Fisheries Research Board of Canada, 34, 1148-1154. https://doi.org/10.1139/f77-172

[17] Harbison, R.D. (1975) Parathion-Induced Toxicity and Phenobarbital-Induced Protection against Paratihion during Prenatal Development. Toxicology and Applied Pharmacology, 32, 482-493. https://doi.org/10.1016/0041-008X(75)90113-1

[18] Oberemm, A. (2000) The Use of a Refined Zebrafish Embryo Bioassay for the Assessment of Aquatic Toxicity. Laboratory Animal, 29, 32-40.

[19] Westerfield, M. (1995) The Zebrafish Book. Guide for the Laboratory Use of Zebrafish (Danio rerio). University of Oregon Press, Eugene.

[20] Jung, M.H., Halter, C., Friesenhengst, A., Walzer, J. and Czerny, T. (2013) Diffusion of Small Molecules into Medaka Embryos Improved by Electroporation. BMC Biotechnology, 13, 53. https://doi.org/10.1186/1472-6750-13-53

[21] Wisk, J.D. and Cooper, K.R. (1990) The Stage Specific Toxicity of 2,3,7,8, Tetrachlorodibenzo-p-Dioxin in the Embryos of the Japanese Medaka (Oryzias latipes). Environmental Toxicology and Chemistry, 9, 1159-1169. https://doi.org/10.1897/1552-8618(1990)9[1159:TSSTOT]2.0.CO;2

[22] Couch, J.A., Winstead, J.T. and Goodman, L.R. (1977) Kepone-Induced Scoliosis and Its Histological Consequences in Fish. Science, 197, 585-587.

[23] Spitsbergen, J.M., Walker, M.K., Oson, J. and Peterson, R.E. (1990) Pathologic Al- 
teration in Early Life Stages of Lake Trout, Salvelinus namaycush, Exposed to 2,3,7,8-tetrachlorodibenzo-p-dioxin as Fertilized Eggs. Aquatic Toxicology, 19, 41-72. https://doi.org/10.1016/0166-445X(91)90027-7 